\title{
Article \\ Binding Properties of Odorant-Binding Protein 4 of Tirathaba rufivena to Areca catechu Volatiles
}

\author{
Xiang Zhou ${ }^{\dagger}$, Zheng Wang ${ }^{\dagger}$, Guangchao Cui, Zimeng Du, Yunlong Qian, Shumei Yang, Minghui Liu \\ and Jixing Guo *D
}

check for

updates

Citation: Zhou, X.; Wang, Z.; Cui, G.;

Du, Z.; Qian, Y.; Yang, S.; Liu, M.;

Guo, J. Binding Properties of

Odorant-Binding Protein 4 of

Tirathaba rufivena to Areca catechu

Volatiles. Plants 2022, 11, 167.

https://doi.org/10.3390/

plants11020167

Academic Editors: Pasquale

Trematerra, Wenwu Zhou

and $\mathrm{Ran} \mathrm{Li}$

Received: 16 November 2021

Accepted: 6 January 2022

Published: 9 January 2022

Publisher's Note: MDPI stays neutral with regard to jurisdictional claims in published maps and institutional affiliations.

Copyright: (c) 2022 by the authors. Licensee MDPI, Basel, Switzerland. This article is an open access article distributed under the terms and conditions of the Creative Commons Attribution (CC BY) license (https:/ / creativecommons.org/licenses/by/ $4.0 /)$.
Key Laboratory of Green Prevention and Control of Tropical Plant Diseases and Pests (Ministry of Education), College of Plant Protection, Hainan University, Haikou 570228, China; zhouxiangdc@aliyun.com (X.Z.); wangzheng56@hainanu.edu.cn (Z.W.); cuiguangchao@hainanu.edu.cn (G.C.); duzimeng@hainanu.edu.cn (Z.D.); hainanuqyl@hainanu.edu.cn (Y.Q.); yangshumei@hainanu.edu.cn (S.Y.); liuminghui@hainanu.edu.cn (M.L.)

* Correspondence: guojixing@hainanu.edu.cn

† These authors contributed equally to this work.

\begin{abstract}
Odorant-binding proteins (OBPs) play a key role in the olfactory system and are essential for mating and oviposition host selection. Tirathaba rufivena, a serious lepidopterous insect pest of the palm area in recent years, has threatened cultivations of Areca catechu in Hainan. Female-biased odorant-binding protein 4 of T. rufivena (TrufOBP4) expression was hypothesized to participate in the process of oviposition host recognition and localization. In this study, we cloned and analyzed the cDNA sequence of TrufOBP4. The predicted mature protein TrufOBP4 is a small, soluble, secretory protein and belongs to a classic OBP subfamily. Fluorescence binding assay results showed that TrufOBP4 had high binding abilities with the host plant volatiles, octyl methoxycinnamate, dibutyl phthalate, myristic acid and palmitic acid. These four components tend to dock in the same binding pocket based on the molecular docking result. The interactions and contributions of key amino acid residues were also characterized. This research provides evidence that TrufOBP4 might participate in the chemoreception of volatile compounds from inflorescences of $A$. catech $u$ and can contribute to the integrated management of T. rufivena.
\end{abstract}

Keywords: odorant-binding proteins; Tirathaba rufivena; binding ability; fluorescence competitive binding assays; molecular docking

\section{Introduction}

The olfactory system of insects plays an important role in behaviors such as host seeking, mating, and oviposition [1]. Insects can acquire information from chemical odorants in the external environment and respond accordingly [2]. The process of odor molecular recognition in insects is a very complex chain reaction [3-5]. The water-soluble carriers are required to transport the lipophilic odorant molecule to receptive membranes. Odorant binding proteins (OBPs) are one of the major protein classes responsible for the binding and transport of water-insoluble compounds through the sensillar lymph [6,7]. OBPs are small and water-soluble proteins, and a typical OBP contains six conserved cysteine residues [8]. An inner hydrophobic pocket used for lipophilic ligand binding is established by the interconnection of disulfide bridges $[9,10]$. In addition, OBPs with more or less than six conserved cysteines have been identified and designated Plus-C OBPs and Minus-C OBPs, respectively $[1,11]$.

The first OBP of lepidopteran insects was reported in the polyphemus moth Antheraea Polyphemus L. (Cramer) (Lepidoptera: Saturniidae) and found to bind sex pheromones [12]. Two specific subclasses, general odorant-binding proteins (GOBPs) and pheromone binding proteins (PBPs), were identified in lepidopteran species based on the distribution pattern in sensilla and similarities of amino acid sequences [13-15]. A large number of GOBPs and PBPs have been identified in the past decade [16-18]. OBPs possess high-specificity 
binding affinity with different plant volatiles and sex pheromones. Based on fluorescence competitive binding assays and computational molecular docking methods, a wide range of volatile binding capabilities have been reported [19]. However, it is still of great significance to characterize both the GOBPs and PBPs from different target insects chosen for study because the insect olfactory system is complex.

Tirathaba rufivena Walker (Lepidoptera: Pyralidae) is an important pest of areca palm, Areca catechu Linn. (Arecales, Arecaceae), in China [20]. This pest has seriously threatened areca nut cultivation in Hainan Province in recent years. Adult females usually lay eggs inside the gap at the base of the inflorescence before it unfolds. Newly hatched larvae penetrate the spathe and feed on the inflorescence. During the flowering and fruiting stages, the larvae can also eat petals and nuts, resulting in reduced yield [20]. Due to the invisibility of pests, the use of chemical pesticides cannot achieve good control effects, and the trees of $A$. catechu are usually tall, increasing the difficulty of pesticide application [21].

Odorant-binding proteins have been considered as pest control targets. Based on analyses of binding affinity, molecular docking and 3D crystal structures, a large number of new powerful semiochemicals have been identified, accelerating the discovery of active chemicals that could be used to manipulate insect behaviors for pest management [22]. Volatiles from areca inflorescence play roles in communication and guiding oviposition host localization [23-25]. The volatile components in areca inflorescence were determined and analyzed by GC-MS [26,27]. The combination of OBP from T. rufivena with semiochemicals is the key process by which T. rufivena finds a suitable host and finishes the reproduction process. Our previous study suggested that TrufOBP4 was highly expressed in female adults. It was hypothesized that TrufOBP4 has a possible functional role in the female-specific odor recognition process. To examine this hypothesis, we cloned the cDNA sequence of TrufOBP4; the binding activities of TrufOBP4 to host plant volatiles of A. catechu were characterized; and key amino acid residues that contributed to their binding interactions were identified. This study will provide evidence that TrufOBP4 might be involved in the chemoreception of host volatile compounds and can successfully contribute to the integrated management of T. rufivena.

\section{Results}

\subsection{Sequence Analysis of TrufOBP4}

Based on the transcriptome data of T. rufivena, the cDNA sequence of TrufOBP4 was cloned and submitted to the NCBI GenBank database (Accession No: OK484430). The TrufOBP4 sequence contained an open reading frame (ORF) of $450 \mathrm{bp}$ and encoded 149 amino acids with a 19 amino acid residue signal peptide at the N-terminus (Figure 1). The molecular mass and acidic isoelectric point of the predicted mature protein were $16.5 \mathrm{kDa}$ and 4.98 , respectively. The hydropathic nature of TrufOBP4 calculated and plotted for each residue showed that the grand average hydropathicity was -0.578 and that the four residue regions were hydrophobic.

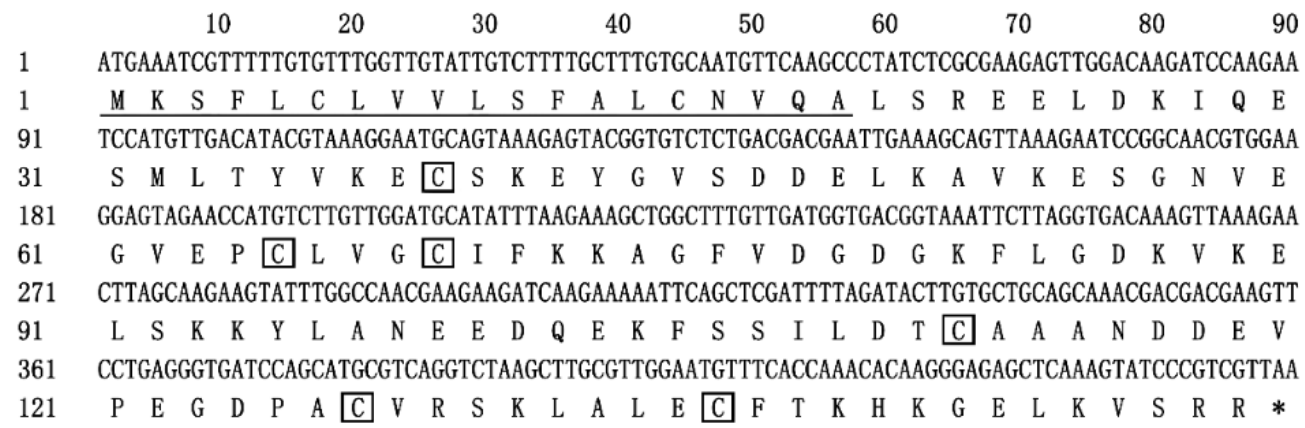

Figure 1. The cDNA and deduced amino acid sequences of TrufOBP4. The predicted signal peptide is indicated by underlining. The conserved Cys sites are indicated in black boxes. The translationtermination codon is marked by an asterisk * 
We conducted an alignment of the amino acid sequence of TrufOBP4 with similar OBPs. The translated amino acid sequence of TrufOBP4 shows approximately $43.85-72.00 \%$ identity with the OBPs of other Pyraloidae insects. TrufOBP4 had the following conserved

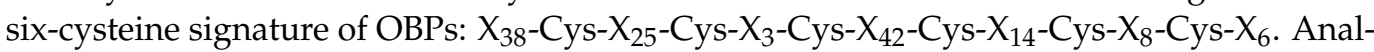
$y$ sis with the Self-Optimized Prediction Method with Alignment (SOPMA) demonstrated that the $\alpha$-helix was the main structure of TrufOBP4 $(63.85 \%)$, in addition to random coils $(25.38 \%), \beta$ turns (8.46\%), and extended strands (2.31\%) (Figure 2$)$. No hydrophobic transmembrane helices were found in TrufOBP4 by ESPript3.0 [28].

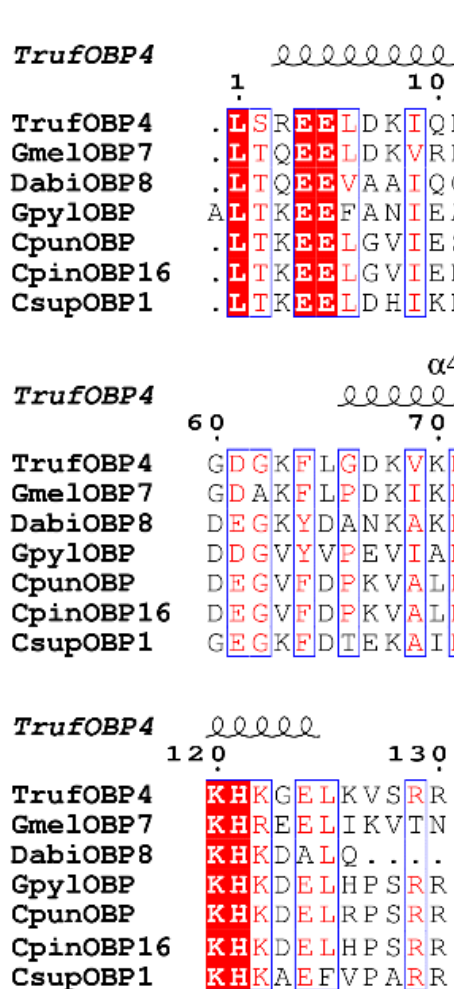

Figure 2. Alignment of TrufOBP4 with OBP genes from other Pyraloidae insect species. Galleria mellonella (Gmel), Dioryctria abietella (Dabi), Glyphodes pyloalis (Gpyl), Conogethes punctiferalis (Cpun), Conogethes pinicolalis (Cpin), and Chilo suppressalis (Csup). The bound cysteines that formed disulfide bridges are marked by green digits at the bottom of sequences.

The 3D structure model was constructed using the crystal structures of $B$. mori pheromone binding protein (20.97\% identity) as a template model. The quality and accuracy of the predicted model were evaluated. The Ramachandran plot showed $91.2 \%$ of residues in the favorable region, and $97.4 \%$ of all residues were in allowed regions (Figure S1A). The Verify 3D results showed that $90.63 \%$ of TrufOBP 4 residues scored above 0.2 (Figure S1B). This protein had a secondary structure consisting of six $\alpha$-helices, which are located between residues Arg22 and Tyr43 ( $\alpha 1)$, Asp47 and Glu55 ( $\alpha 2)$, Pro64 and Lys74 ( $\alpha 3)$, Gly85 and Tyr95 ( $\alpha 4)$, Glu99 and Ala114 ( $\alpha 5)$, and Ala126 and Glu143 ( $\alpha 6)$ (Figure 3A). The framework of helices was stabilized by three highly conserved internal disulfide bridges with six cysteine residues, Cys39-Cys69, Cys65-Cys136 and Cys112-Cys127. A pocket surrounded by hydrophobic residues was observed in the form of a tunnel occupying approximately $223 \AA^{3}$ (Figure 3B).

\subsection{Phylogenetic Tree Construction of TrufOBP4}

A phylogenetic tree was constructed with TrufOBP4 and nineteen OBP sequences of other species of Lepidoptera to assess evolutionary relationships among the proteins. TrufOBP4 was clustered with GmelOBP7 (QEI46791.1), DabiOBP8 (QQG64121.1), OfurOBP3 
(BAV56790.1), GpylOBP (QIJ45744.1), and CsupOBP (AGM38610.1) in the Pyraloidea superfamily, in which TrufOBP4 was close to the protein GmelOBP7 (QEI46791.1) with 72.00\% similarity (Figure 4).

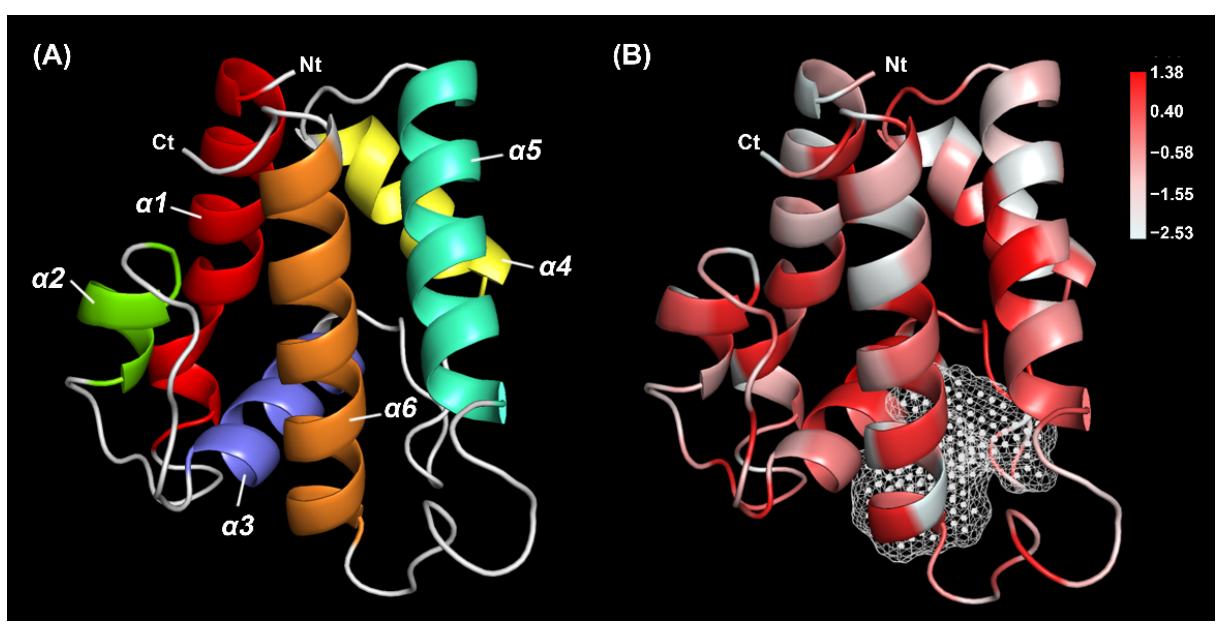

Figure 3. Three-dimensional structure model of TrufOBP4. (A) The 3D structures of TrufOBP4 with six helices labeled and colored by the rainbow. (B) The hydrophobic profiles and predicted binding pocket of TrufOBP4. The levels of hydrophobic value were illustrated by a gradient color scale, and the binding pocket was marked by white dots. Nt: N-terminus, Ct: C-terminus.

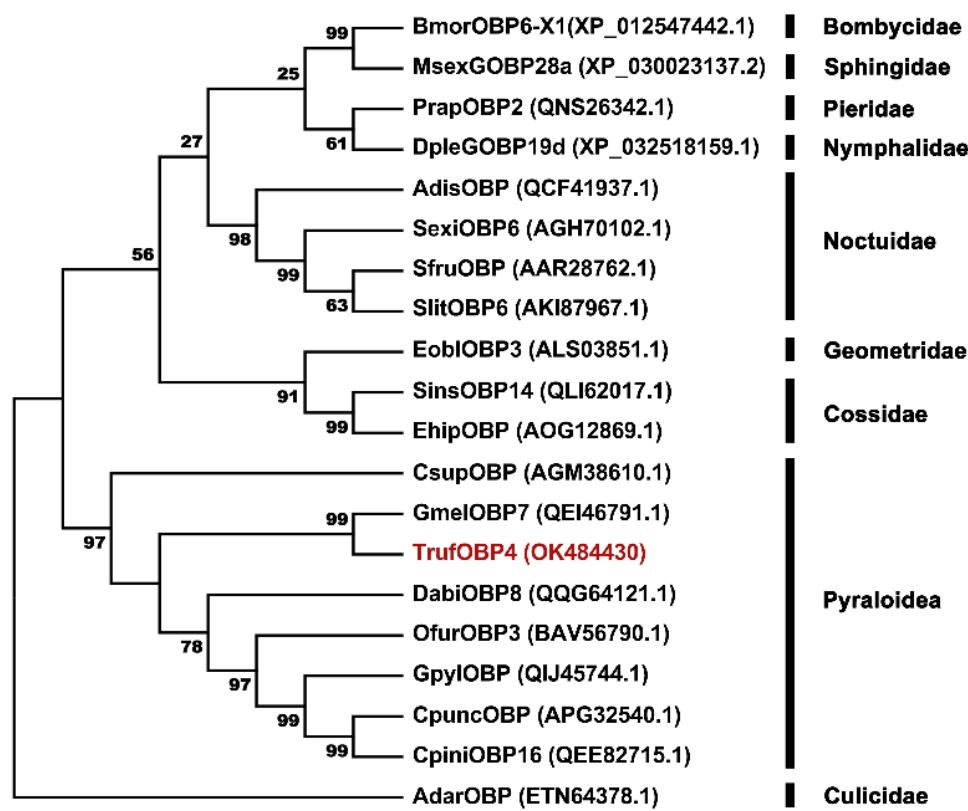

Figure 4. Phylogenetic tree of TrufOBP4 amino acid sequences with OBPs from other lepidopteran and dipteran insect species. The tree was constructed using the neighbor-joining method with a bootstrap of 1000 replicates. AdarOPB from Anopheles darlingi (Diptera: Culicidae) were used as the outgroup to root the tree. Anopheles darlingi (Adar), Athetis dissimilis (Adis), Bombyx mori (Bmor), Conogethes pinicolalis (Cpin), Conogethes punctiferalis (Cpun), Chilo suppressalis (Csup), Dioryctria abietella (Dabi), Danaus plexippus (Dple), Ectropis obliqua (Eobl), Eogystia hippophaecolus (Ehip), Galleria mellonella (Gmel), Glyphodes pyloalis (Gpyl), Manduca sexta (Msex), Ostrinia furnacalis (Ofur), Pieris rapae (Prap), Spodoptera exigua (Sexi), Spodoptera frugiperda (Sfru), Streltzoviella insularis (Sins), and Spodoptera litura (Slit). 


\subsection{Expression and Purification of Recombinant TrufOBP4}

The recombinant TrufOBP4 protein was successfully expressed as a soluble protein in the supernatant of E. coli BL21 (DE3) after induction with isopropyl $\beta$-D-1thiogalactopyranoside (IPTG). After ultrasonication, the proteins were purified using Ni-NTA resin affinity chromatography. Then, the His-tag was removed by enterokinase. After confirmation by SDS-PAGE, the purified OBP proteins were further used to test the binding properties (Figure 5).
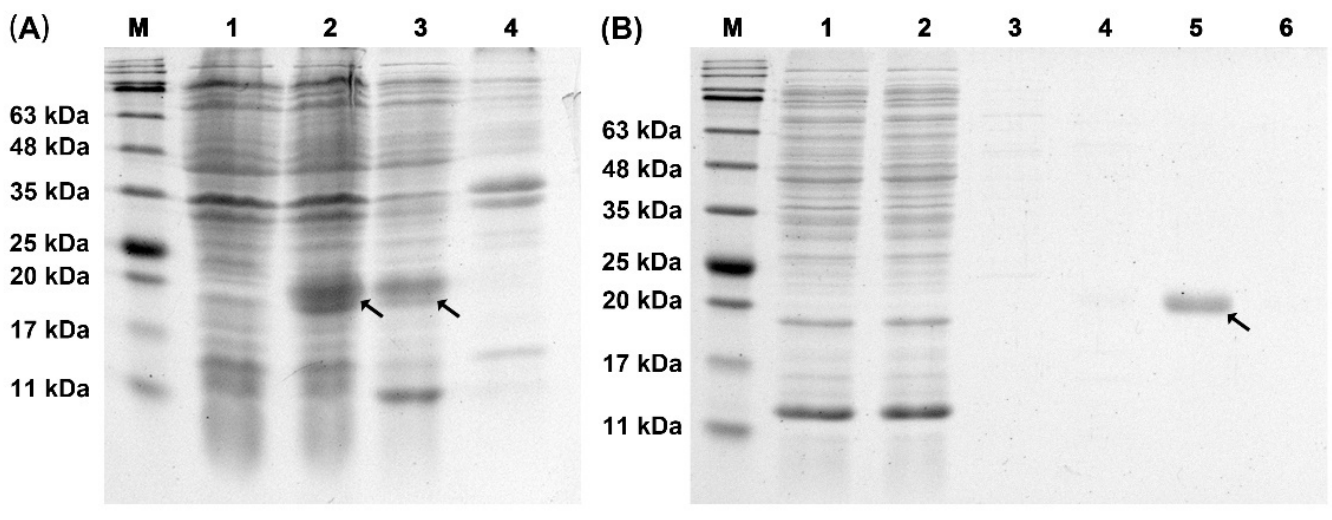

Figure 5. Expression and purification of recombinant protein TrufOBP4 analyzed by SDS-PAGE. (A) SDS-PAGE analysis of the expression of recombinant TrufOBP4. M: Protein molecular weight marker. 1: Protein sample without IPTG induction; 2: Protein sample with $0.5 \mathrm{mM}$ IPTG induction. 3: The supernatant of protein sample with $0.5 \mathrm{mM} \mathrm{IPTG}$ induction after ultrasonic crushing. 4: The precipitation of protein sample with $0.5 \mathrm{mM}$ IPTG induction after ultrasonic crushing. (B) SDS-PAGE analysis of the purification of recombinant TrufOBP4. M: Protein molecular weight marker. 1: Protein fluid after flowing through the column. 2-6: Protein samples were obtained with imidazole elution at different concentrations $(0,40,100,200$ and $1000 \mathrm{mmol} / \mathrm{L}$ imidazole). The target protein was marked by an arrow.

\subsection{Fluorescence Competitive Binding Assays}

Using competitive binding assays, we tested nineteen volatiles from areca inflorescences as competitors. The fluorescent reporter 1-phenyl-1-naphthylamine(1-NPN) was employed to test the binding affinity constants with TrufOBP4. Scatchard plots were analyzed to calculate the dissociation constant. The TrufOBP4 binds to 1-NPN with a $\mathrm{K} 1-\mathrm{NPN}$ of $6.79 \mu \mathrm{M}$ (Figure 6). The K1-NPN value was then used to calculate the Ki values of nineteen volatiles with TrufOBP4. The competitive fluorescence binding curves showed that most of the ligands could reduce the relative fluorescence intensity of the TrufOBP4/1-NPN complex, indicating that TrufOBP4 could bind to all nineteen volatiles. The compounds octyl methoxycinnamate, dibutyl phthalate, myristic acid and palmitic acid had high binding affinities to TrufOBP6 with Ki values of 1.16, 4.61, 3.52 and $5.28 \mu \mathrm{M}$, respectively (Table 1 ). 
(A)

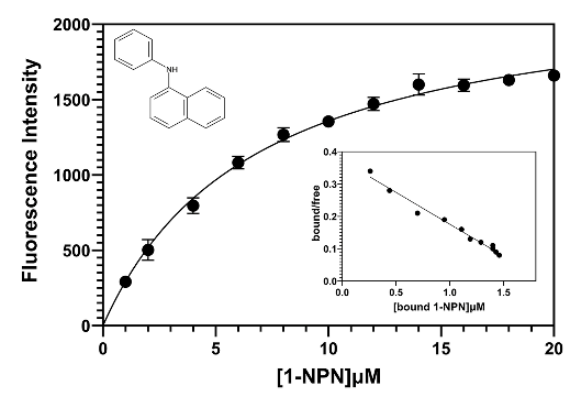

(B)
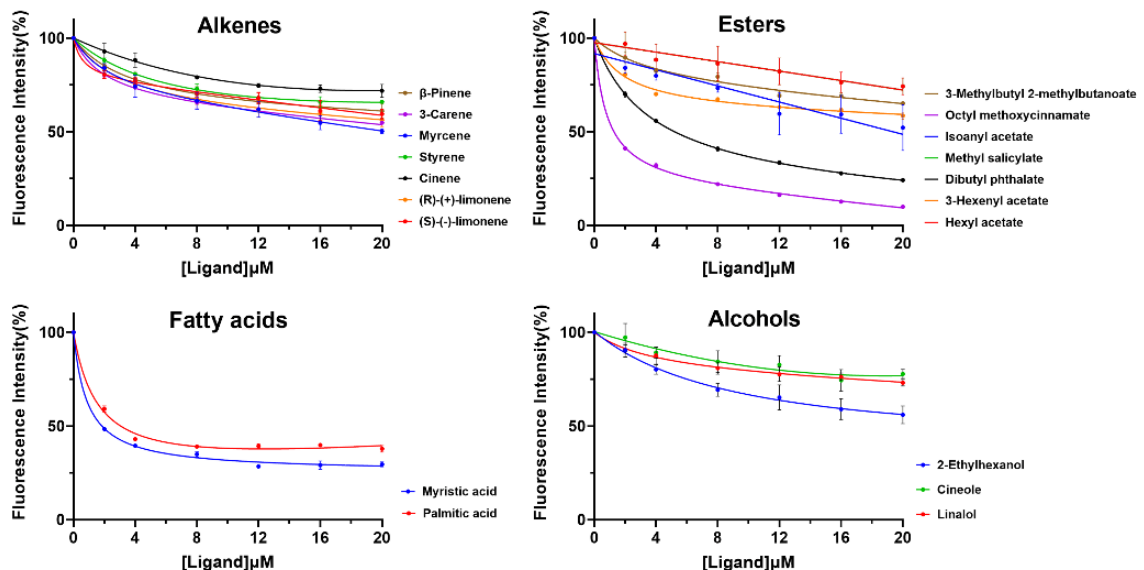

Figure 6. Competitive fluorescence ligand-binding assay of TrufOBP4 to volatiles. (A) Binding curve and relative Scatchard plot of TrufOBP4 and 1-NPN. (B) Competitive binding curves of TrufOBP4 with alkene, esters, fatty acids and alcohols.

Table 1. Binding affinities of different ligands to TrufOBP4.

\begin{tabular}{cccccc}
\hline No. & Ligands & Formula & CAS No\# & IC50 $(\mu \mathbf{M})$ & Ki $(\mu \mathbf{M})$ \\
\hline 1 & $(\mathrm{R})-(+)$-limonene & $\mathrm{C}_{10} \mathrm{H}_{16}$ & $5989-27-5$ & 25.32 & 22.07 \\
2 & (S)-(-)-Limonene & $\mathrm{C}_{10} \mathrm{H}_{16}$ & $5989-54-8$ & 33.36 & 29.08 \\
3 & 2-Ethylhexanol & $\mathrm{C}_{8} \mathrm{H}_{18} \mathrm{O}$ & $104-76-7$ & 24.08 & 20.99 \\
4 & Octyl methoxycinnamate & $\mathrm{C}_{18} \mathrm{H}_{26} \mathrm{O}_{3}$ & $83834-59-7$ & 1.33 & 1.16 \\
5 & 3-Carene & $\mathrm{C}_{10} \mathrm{H}_{16}$ & $13466-78-9$ & 22.41 & 19.53 \\
6 & Methyl salicylate & $\mathrm{C}_{8} \mathrm{H}_{8} \mathrm{O}_{3}$ & $119-36-8$ & $>50$ & $>50$ \\
7 & Cinene & $\mathrm{C}_{10} \mathrm{H}_{16}$ & $138-86-3$ & $>50$ & $>50$ \\
8 & Cineole & $\mathrm{C}_{10} \mathrm{H}_{18} \mathrm{O}$ & $470-82-6$ & $>50$ & $>50$ \\
9 & 3-Hexenyl acetate & $\mathrm{C}_{8} \mathrm{H}_{14} \mathrm{O}_{2}$ & $3681-71-8$ & 46.59 & 40.61 \\
10 & Dibutyl phthalate & $\mathrm{C}_{16} \mathrm{H}_{22} \mathrm{O}_{4}$ & $84-74-2$ & 5.29 & 4.61 \\
11 & Hexyl acetate & $\mathrm{C}_{8} \mathrm{H}_{16} \mathrm{O}_{2}$ & $142-92-7$ & $>50$ & $>50$ \\
12 & Isoanyl acetate & $\mathrm{C}_{7} \mathrm{H}_{14} \mathrm{O}_{2}$ & $123-92-2$ & 24.11 & 21.02 \\
13 & 3-Methylbutyl & $\mathrm{C}_{10} \mathrm{H}_{20} \mathrm{O}_{2}$ & $27625-35-0$ & $>50$ & 45.83 \\
14 & 2-methylbutanoate & $\mathrm{C}_{10} \mathrm{H}_{18} \mathrm{O}$ & $78-70-6$ & $>50$ & $>50$ \\
15 & Linalol & $\mathrm{C}_{10} \mathrm{H}_{16}$ & $123-35-3$ & 19.80 & 17.26 \\
16 & Myrcene & $\mathrm{C}_{14} \mathrm{H}_{28} \mathrm{O}_{2}$ & $544-63-8$ & 4.04 & 3.52 \\
17 & Myristic acid & $\mathrm{C}_{16} \mathrm{H}_{32} \mathrm{O}_{2}$ & $57-10-3$ & 6.06 & 5.28 \\
18 & Palmitic acid & $\mathrm{C}_{8} \mathrm{H}_{8}$ & $100-42-5$ & 41.50 & 36.17 \\
19 & Styrene & $\mathrm{C}_{10} \mathrm{H}_{16}$ & $18172-67-3$ & 33.29 & 29.02 \\
\hline
\end{tabular}

Note: " $>50$ " for the IC50 and Ki means that IC50 or Ki cannot be accurately calculated with the ligand concentration range tested in the assay.

\subsection{Molecular Docking}

To further investigate the binding mode of TrufOBP4 with the chemicals and validate the results of the ligand-binding assay, the tested ligands with the highest binding ability (Ki below $10 \mu \mathrm{M}$ ) were chosen and docked against TrufOBP4. These four compounds, octyl methoxycinnamate, dibutyl phthalate, myristic acid and palmitic acid, exhibited good interactions against TrufOBP4 with binding energies of $-5.24,-5.91,-5.69$ and -5.11 , respectively. All compounds tended to dock in the same binding pocket. Van der Waals interactions, a hydrogen bond (H-bond) and covalent interactions were found between TrufOBP4 and the compounds (Figure 7). 
(A) Octyl methoxycinnamate

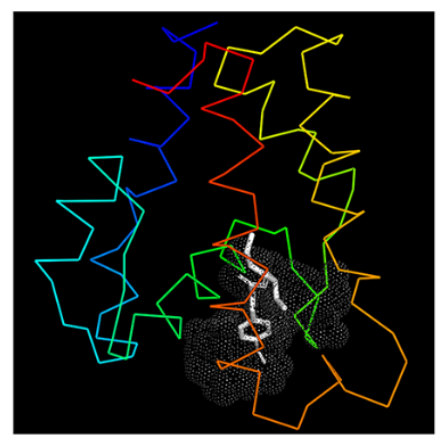

(C) Myristic acid (n)
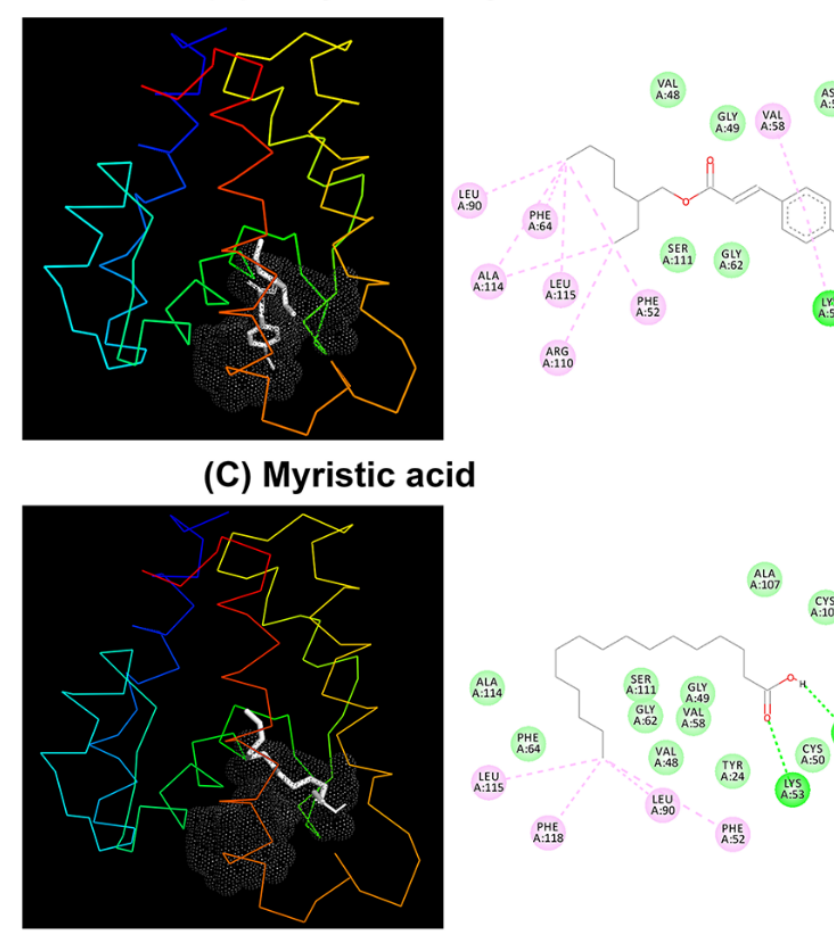

(B) Dibutyl phthalate
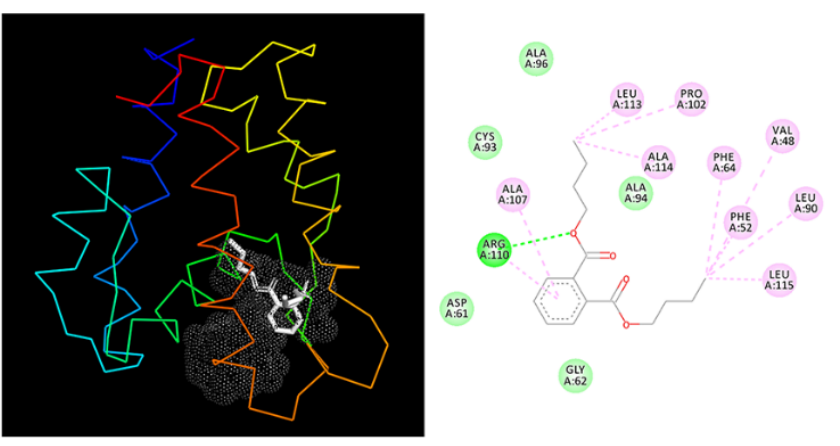

(D) Palmitic acid

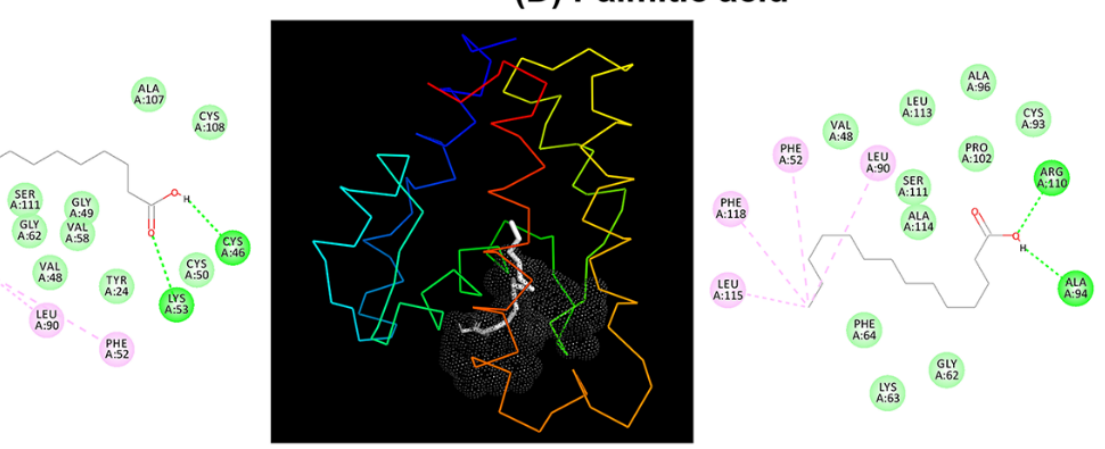

Figure 7. Molecular docking of TrufOBP4 with ligands. (A) 3D predicted interaction view of binding pocket (left) and 2D predicted interaction (right) of TrufOBP4 and octyl methoxycinnamate. (B) 3D predicted interaction view of binding pocket (left) and 2D predicted interaction (right) of TrufOBP4 and dibutyl phthalate. (C) 3D predicted interaction view of binding pocket (left) and 2D predicted interaction (right) of TrufOBP4 and myristic acid. (D) 3D predicted interaction view of binding pocket (left) and 2D predicted interaction (right) of TrufOBP4 and palmitic acid.

Octyl methoxycinnamate showed van der Waals interactions toward Val48, Gly49, Asp59, Gly62 and Ser111, while it formed covalent interactions with Phe52, Val58, Phe64, Leu90, Arg110, Ala114 and Leu115. Only Lys53 formed an H-bond. Dibutyl phthalate bound against Asp61, Gly62, Cys93, Ala94 and Ala96 through van der Waals interactions and Val48, Phe52, Phe64, Leu90, Pro102, Ala107, Ala114, Leu113 and Leu115 through covalent interactions. Only Arg110 formed an H-bond. Myristic acid formed an H-bond with Cys46 and Lys53. Phe52, Leu90, Leu115 and Phe118 were involved in covalent bonding, while Tyr24, Val48, Gly49, Cys50, Val58, Gly62, Phe64, Ala107, Cys108, Ser111 and Ala114 were bound through van der Waals interactions. During palmitic acid and TrufOBP4 binding, Phe52, Leu90, Leu115 and Phe118 were involved in covalent bonding, while Val48, Gly62, Phe64, Cys93, Ala96, Pro102, Ser111, Leu113 and Ala114 were bound through van der Waals interactions, and Ala94 and Arg110 formed an H-bond.

\section{Discussion}

The insect olfaction system allows insects to recognize and track volatile cues from host plants and mates, avoid toxic compounds and evade their predators [19]. The OBPs play an important role in olfactory sensation and are essential for binding odorant molecules and facilitating their transport through the aqueous neuronal environment [29]. It was suggested that TrufOBP4, one of the most abundant OBPs expressed in females, could participate in the female-specific olfactory recognition process. In this study, we cloned and analyzed the cDNA sequence of TrufOBP4. The predicted mature protein TrufOBP4 was a small, soluble, secretory protein with a molecular mass of $16.5 \mathrm{kDa}$ and 19 amino acid residue signal peptides at the N-terminus. The hydropathic nature of TrufOBP4 was very similar to that of other insect OBPs. The sequences of TrufOBP4 displayed the 
conserved "OBP sequence motif of Lepidoptera", and the conserved motif was $\mathrm{C}_{1}-\mathrm{X}_{25-30^{-}}$ $\mathrm{C}_{2}-\mathrm{X}_{3}-\mathrm{C}_{3}-\mathrm{X}_{36-42}-\mathrm{C}_{4}-\mathrm{X}_{8-14}-\mathrm{C}_{5}-\mathrm{X}_{8}-\mathrm{C}_{6}[30,31]$. Three pairs of disulfide bridges were formed by six conserved cysteines, which demonstrated that TrufOBP4 could belong to classic OBP subfamilies. Amino acid sequence analysis of TrufOBP4 with similar OBPs indicated that TrufOBP4 shared high sequence identity with other Pyraloidae insects. TrufOBP4 was clustered with GmelOBP7 in the phylogenetic tree, which was consistent with the highest sequence similarity between them. This indicated that these homologous genes are evolutionarily conserved and play similar biological roles in olfaction. The 3D structure model showed that TrufOBP4 had a secondary structure consisting of six $\alpha$-helices and internal cavity, which were stabilized by three highly conserved internal disulfide bridges.

Data from fluorescence binding assays showed that TrufOBP4 is specifically bound to octyl methoxycinnamate, dibutyl phthalate, myristic acid and palmitic acid with high binding affinities. The docking result also confirmed the interactions of these four compounds with TrufOBP4. The binding capacity of TrufOBP4 with host plant volatiles was attributed to van der Waals interactions, hydrogen bonds, and covalent interactions ( $\mathrm{Pi}$ alkyls). Hydrophobic residues, such as V48, F52, G62, F64, L90, A114, and L115, which were highly overlapped, were conserved, contributing to the interaction with odors rather than hydrophilic residues. Previous studies in Apolygus lucorum (Hemiptera: Miridae) also showed that mutants of hydrophobic residues decreased or completely abolished binding affinities to ligands. These amino acid residues play vital roles in the formation of the interaction between a protein and hydrophobic ligand. The specific role of these residues needs further study through a site-directed mutagenesis technique [12,29,32-34].

The high binding affinities of octyl methoxycinnamate, dibutyl phthalate, myristic acid and palmitic acid to the female-biased expressed OBPs of adult T. rufivena suggested an essential role for these odor molecules in the chemoreception of volatiles from the host plant $A$. catechu. Dibutyl phthalate was reported to be a common component of many plant volatiles [35]. Many studies have demonstrated that dibutyl phthalate can influence the behavior of insects $[24,25,36]$. For example, this compound had an attractive effect on Holotrichia oblita Faldermann (Coleoptera: Scarabaeidae) but was repellent to Rhyzopertha dominica (Coleopera: Bostrichidae) and Tribolium castaneum (Coleoptera: Tenebrionidae) [35]. In addition, a study indicated that mating rates were significantly increased by the stimulation of Hyphantria cunea (Lepidoptera: Arctiidae) with dibutyl phthalate [24]. The OBPs of many insect species were also reported to have a high binding affinity to dibutyl phthalate. Some studies in lepidopteran demonstrated that AipsGOBP2 of Agrotis ipsilon (Lepidoptera: Noctuidae) and EoblGOBP2 of Ectropis obliqua (Lepidoptera: Geometridae) show an outstanding binding affinity to dibutyl phthalate [36,37]. In addition, the high binding affinities of OBPs with dibutyl phthalate were observed in other species [25]; for instance, the ability to perceive dibutyl phthalate of $H$. oblita females decreased once HoblOBP7 expression was knocked down $[25,36]$. Therefore, we propose that dibutyl phthalate can also influence the behavior of T. rufivena.

Myristic acid and palmitic acid were also proven to be among the best ligands for TrufOBP4. Some researchers have reported that OBP has a high binding ability with fatty acids [38]. The MbraPBP1 of Mamestra brassicae (Lepidoptera: Noctuidae) and LsatOBP1 of Liriomyza sativae Blanchard (Diptera: Agromyzidae) bind well to palmitic acid [12,39]. Fatty acids are not only basic components of insects and plants but also play important physiological roles in insects. The PregOBP56a, which showed a high binding affinity with palmitic, stearic, oleic, and linoleic acids, was proposed to solubilize these fatty acids and deliver them to the midgut in the reproductive process [38]. However, the olfactory peripheral coding mechanism of myristic acid and palmitic acid and its function in the physiological process still need further study. 


\section{Materials and Methods}

\subsection{Insect Rearing}

Larvae of T. rufivena were collected from the host plant areca palms in Qionghai County $\left(19.23^{\circ} \mathrm{N}, 110.47^{\circ} \mathrm{E}\right)$, Hainan province, China. The larvae were reared in the laboratory under environment conditions at $28 \pm 1{ }^{\circ} \mathrm{C}$, with a relative humidity of $80 \pm 10 \%$ and a photoperiod of 14:10 (L:D) with an artificial diet.

\subsection{RNA Extraction and $c D N A$ Synthesis}

Antennas from ten female adults (first generation) were collected and immediately frozen in liquid nitrogen. Total RNA was extracted using TRIzol reagent (Invitrogen, Carlsbad, USA). RNA integrity was determined by $1 \%$ agarose gels. RNA quantity and purification were checked using a NanoPhotometer Spectrophotometer (Implen, CA, USA). First-strand cDNA was synthesized by a HiScript ${ }^{\circledR}$ II Reverse Transcriptase synthesis kit (Vazyme, Nanjing, China) according to the manufacturer's instructions.

\subsection{Cloning and Sequencing Analysis}

Specific primers were designed and synthesized by Sangon Biotech Company (Shanghai, China) based on the nucleotide sequence of TrufOBP4. The sense primer was 5TGTGACGCTTTACACTAA-3, and the antisense primer was 5AGTCGTCTAATCAGGAAA-3. PCR studies were performed using Phanta Max SuperFidelity DNA Polymerase (Vazyme, Jiangsu, China). The amplification of the TrufOBP4 cDNA fragment was performed on a Veriti Thermal Cycler (AB, CA, USA) with the following PCR procedure: $94{ }^{\circ} \mathrm{C}$ for $5 \mathrm{~min}$; 30 cycles at $94^{\circ} \mathrm{C}$ for $30 \mathrm{~s}$, at $60^{\circ} \mathrm{C}$ for $30 \mathrm{~s}$, and at $72{ }^{\circ} \mathrm{C}$ for $30 \mathrm{~s}$; and $72{ }^{\circ} \mathrm{C}$ for $10 \mathrm{~min}$. The colonies were sequenced by Sangon Biotech Company (Shanghai, China). The cDNA sequence and deduced amino acid sequence of TrufOBP4 were analyzed using DNAMAN (Lynnon Biosoft, CA, USA). The putative Nterminal signal peptides were predicted using the SignalP V4.1 program (https: / / services. healthtech.dtu.dk/service.php?SignalP-4.1 (accessed on 10 January 2021)). The chemical and physical properties, secondary structure and hydrophobicity scales of TrufOBP4 were predicted using the online program tools ProtParam (https:/ / web.expasy.org/protparam/ (accessed on 10 January 2021)), SOPMA (https:/ / npsa-prabi.ibcp.fr/cgi-bin/ (accessed on 10 January 2021)), ProtScale (https: / / web.expasy.org/protscale/ (accessed on 15 January 2021)) and ESPript3.0 software [28]. The larger the hydrophobic value, the stronger the hydrophobicity of this amino acid [40]. ClustalW and MEGA 5.2 were employed for multiple alignments and the phylogenetic tree construction of TrufOBP4 with similar OBPs of other insect species using the neighbor-joining method with a No. of differences model and a pairwise deletion of gaps.

\subsection{Expression and Purification of Recombinant TrufOBP4}

The cDNA sequence of TrufOBP4-removed signal peptides was amplified using designed primers. The enzymatic digestion sites were designated EcoRI and NotI. The sense primer was 5-CGGAATTCCTATCTCGCGAAGAGTTGG-3 (the EcoRI restriction site is underlined), and the antisense primer was 5-AAATATGCGGCCGC ACGACGGGATACTTTGAGCT-3 (the NotI restriction site is underlined). The purified PCR product and pET-32a (+) vector (Solarbio, Beijing, China) were ligated and transformed into E. coli BL21 (DE3) cells (AxyGen, Shanghai, China). Cells of E. coli were cultured until the OD600 value reached 0.6 , while $\beta$-D-1-thiogalactopyranoside (IPTG) was added to the LB medium at a final concentration of $0.5 \mathrm{mM}$. The bacterial cultures were shaken continuously at $200 \mathrm{rpm}$ at $30^{\circ} \mathrm{C}$ for $6 \mathrm{~h}$ to induce recombinant TrufOBP4 protein. The suspension was crushed by sonication and then separated into supernatant and sediment by centrifugation (6000 rpm, $10 \mathrm{~min}, 4^{\circ} \mathrm{C}$ ). Then, pET-32a (+)-TrufOBP4 was purified and desalted using Ni Sepharose 6FF (Solarbio, Beijing, China) and Amicon ${ }^{\circledR}$ Ultra-4 3K centrifugal filters (Merck Millipore, Darmstadt, Germany), respectively. The size and purity of the recombinant protein were analyzed with $12 \%$ SDS-PAGE. 


\subsection{Fluorescence Competitive Binding Assays}

The binding ability of purified recombinant OBPs was evaluated with an F-7000 fluorescence spectrometer (Hitachi, Tokyo, Japan) [41,42]. The fluorescence spectra were recorded between 360 and $650 \mathrm{~nm}$. N-Phenyl-1-naphthylamine (1-NPN) was selected as the fluorescent reporter and dissolved in chromatographic methanol at a concentration of $1 \mathrm{mM}$. The purified TrufOBP4 was dissolved in $50 \mathrm{mM}$ Tris- $\mathrm{HCl}(\mathrm{pH} 7.4)$ at a final concentration of $2 \mu \mathrm{M}$. The data for the 1-NPN-TrufOBP4 complex formation were obtained by titration of $2 \mu \mathrm{M}$ protein with increasing concentrations (2 to $20 \mu \mathrm{M})$ of $1-\mathrm{NPN}$. The dissociation constant of 1-NPN (K $1-\mathrm{NPN}$ ) was calculated using Prism 8 (GraphPad Software, San Diego, USA). To evaluate the binding ability of TrufOBP4 with volatiles, 1-NPN was replaced with different odor compounds from the TrufOBP4/1-NPN complex. The odor ligands were dissolved in chromatographic methanol at a concentration of $1 \mathrm{mM}$ and added as aliquots to the protein solution. The spectral results were obtained, and the dissociation constants of the competitors were calculated with the formula: $\mathrm{Ki}=\mathrm{IC} 50 /\left(1+[1-\mathrm{NPN}] / \mathrm{K}_{1-\mathrm{NPN}}\right)$, where 1-NPN represents the free concentration of 1-NPN, and IC50 represents the ligand concentration displacing $50 \%$ of the fluorescent reporter [43].

\subsection{Structure Modelling and Molecular Docking}

The predicted three-dimensional structure (3D) of TrufOBP4 was modelled using the SWISS-MODEL prediction algorithm (https:/ / swissmodel.expasy.org/ (accessed on 5 April 2021)) with the crystal structure of BmorPBP (PDB: 2p70.1. A) selected as the template [44]. The three-dimensional (3D) conformer structures of candidate volatiles were downloaded from the chemical compound databases ZINC (https:/ / zinc.docking.org/ (accessed on 5 March 2021)) and PubChem (https:/ / pubchem.ncbi.nlm.nih.gov/ (accessed on 5 March 2021)). The 3D model quality assessment was performed using SAVES v6.0 (https:/ / saves.mbi.ucla.edu (accessed on 9 April 2021)). The POCASA 1.1 program (https: //g6altair.sci.hokudai.ac.jp/g6/service/pocasa/ (accessed on 5 April 2021)) was used to make a pocket prediction. AutoDock (Molecular Graphics Laboratory, La Jolla, CA, USA) was used to find the potential binding mode between TrufOBP4 and ligands. The binding affinity score was calculated based on the potential energy changes around the binding pocket during the protein-ligand interaction. A lower score corresponded to a stronger binding ability. Visual structure analysis was carried out by PYMOL Viewer (http:/ / www.pymol.org/ (accessed on 20 April 2021)) and Discovery Studio visualizer (BIOVIA, San Diego, CA, USA).

\section{Conclusions}

In this study, the cDNA sequence of TrufOBP4, a female-biased expressed OBP, was cloned and analyzed. The TrufOBP4 showed high binding abilities with the host plant volatiles, octyl methoxycinnamate, dibutyl phthalate, myristic acid and palmitic acid based on fluorescence binding assays and molecular docking. The interactions and contributions of key amino acid residues were also characterized. This study provides evidence that TrufOBP4 might be involved in the chemoreception of host volatile compounds, and that a potential ecological-based trapping method can be developed by utilizing these common volatiles.

Supplementary Materials: The following are available online at https:/ / www.mdpi.com/article/ 10.3390/plants11020167/s1, Figure S1: The model quality of TrufOBP4 evaluated by Procheck and Verify_3D.

Author Contributions: Conceptualization, X.Z.; Data curation, X.Z. and Z.W.; Funding acquisition, X.Z. and J.G.; Methodology, X.Z. and Z.W.; Resources, G.C., Z.D., Y.Q. and M.L.; Software, S.Y.; Writing-original draft, X.Z. and J.G. All authors have read and agreed to the published version of the manuscript. 
Funding: This work was funded by Natural Science Foundation of Hainan Province of China (2019RC136), the Startup Funding of Hainan University (No. KYQD (ZR)1879) and the Hainan Major Research Project of Science and Technology (ZDKJ201817).

Institutional Review Board Statement: Not applicable.

Informed Consent Statement: Not applicable.

Data Availability Statement: All data included in the main text and Supplementary Materials.

Conflicts of Interest: The authors declare no conflict of interest.

\section{References}

1. Zhou, J.J. Odorant-binding proteins in insects. Vitam. Horm. 2010, 83, 241-272. [CrossRef] [PubMed]

2. Hansson, B.S.; Stensmyr, M.C. Evolution of insect olfaction. Neuron 2011, 72, 698-711. [CrossRef] [PubMed]

3. Mohapatra, P.; Menuz, K. Molecular profiling of the Drosophila antenna reveals conserved genes underlying olfaction in Insects. G3-Genes Genomes Genet. 2019, 9, 3753-3771. [CrossRef] [PubMed]

4. Sachse, S.; Krieger, J. Olfaction in insects. e-Neuroforum 2011, 2, 49. [CrossRef]

5. Wei, H.; Tan, S.; Li, Z.; Li, J.; Moural, T.W.; Zhu, F.; Liu, X. Odorant degrading carboxylesterases modulate foraging and mating behaviors of Grapholita molesta. Chemosphere 2021, 270, 128647. [CrossRef] [PubMed]

6. Pelosi, P.; Zhou, J.J.; Ban, L.P.; Calvello, M. Soluble proteins in insect chemical communication. Cell. Mol. Life Sci. 2006, 63, 1658-1676. [CrossRef]

7. Yang, K.; Liu, Y.; Niu, D.J.; Wei, D.; Li, F.; Wang, G.R.; Dong, S.L. Identification of novel odorant binding protein genes and functional characterization of OBP8 in Chilo suppressalis (Walker). Gene 2016, 591, 425-432. [CrossRef] [PubMed]

8. Pelosi, P.; Iovinella, I.; Felicioli, A.; Dani, F.R. Soluble proteins of chemical communication: An overview across arthropods. Front. Physiol. 2014, 5, 320. [CrossRef] [PubMed]

9. Zhou, X.; Guo, J.; Zhang, M.; Bai, C.; Wang, Z.; Li, Z. Antennal transcriptome analysis and candidate olfactory genes in Crematogaster rogenhoferi. Bull. Entomol. Res. 2021, 111, 464-475. [CrossRef] [PubMed]

10. Zhou, J.; Zhang, N.; Wang, P.; Zhang, S.; Li, D.; Liu, K.; Wang, G.; Wang, X.; Ai, H. Identification of host-plant volatiles and characterization of two novel general odorant-binding proteins from the legume pod borer, Maruca vitrata Fabricius (Lepidoptera: Crambidae). PLoS ONE 2015, 10, e0141208.

11. Khuhro, S.A.; Liao, H.; Dong, X.-T.; Yu, Q.; Yan, Q.; Dong, S.-L. Two general odorant binding proteins display high bindings to both host plant volatiles and sex pheromones in a pyralid moth Chilo suppressalis (Lepidoptera: Pyralidae). J. Asia-Pac. Entomol. 2017, 20, 521-528. [CrossRef]

12. Campanacci, V.; Krieger, J.; Bette, S.; Sturgis, J.N.; Lartigue, A.; Cambillau, C.; Breer, H.; Tegoni, M. Revisiting the specificity of Mamestra brassicae and Antheraea polyphemus pheromone-binding proteins with a fluorescence binding assay. J. Biol. Chem. 2001, 276, 20078-20084. [CrossRef]

13. Gong, D.P.; Zhang, H.J.; Zhao, P.; Xia, Q.Y.; Xiang, Z.H. The odorant binding protein gene family from the genome of silkworm, Bombyx mori. BMC Genom. 2009, 10, 332. [CrossRef]

14. Gu, S.H.; Sun, L.; Yang, R.N.; Wu, K.M.; Guo, Y.Y.; Li, X.C.; Zhou, J.J.; Zhang, Y.J. Molecular characterization and differential expression of olfactory genes in the antennae of the black cutworm moth Agrotis ipsilon. PLoS ONE 2014, 9, e103420. [CrossRef]

15. Zhang, T.T.; Wang, W.X.; Gu, S.H.; Zhang, Z.D.; Wu, K.M.; Zhang, Y.J.; Guo, Y.Y. Structure, binding characteristics, and 3D model prediction of a newly identified odorant-binding protein from the cotton bollworm, Helicoverpa armigera (Hübner). J. Integr. Agric. 2012, 11, 430-438. [CrossRef]

16. Vogt, R.G.; Grosse-Wilde, E.; Zhou, J.J. The Lepidoptera odorant binding protein gene family: Gene gain and loss within the GOBP/PBP complex of moths and butterflies. Insect Biochem. Mol. Biol. 2015, 62, 142-153. [CrossRef]

17. Tang, B.; Tai, S.; Dai, W.; Zhang, C. Expression and functional analysis of two odorant-binding proteins from Bradysia odoriphaga (Diptera: Sciaridae). J. Agric. Food Chem. 2019, 67, 3565-3574. [CrossRef]

18. Gong, Z.J.; Zhou, W.W.; Yu, H.Z.; Mao, C.G.; Zhang, C.X.; Cheng, J.A.; Zhu, Z.R. Cloning, expression and functional analysis of a general odorant-binding protein 2 gene of the rice striped stem borer, Chilo suppressalis (Walker) (Lepidoptera: Pyralidae). Insect. Mol. Biol. 2009, 18, 405-417. [CrossRef]

19. Jing, D.; Zhang, T.; Prabu, S.; Bai, S.; He, K.; Wang, Z. Molecular characterization and volatile binding properties of pheromone binding proteins and general odorant binding proteins in Conogethes pinicolalis (Lepidoptera: Crambidae). Int. J. Biol. Macromol. 2020, 146, 263-272. [CrossRef]

20. Zhong, B.; Lv, C.; Qin, W. Preliminary study on biology and feeding capacity of Chelisoches morio (Fabricius) (Dermaptera: Chelisochidae) on Tirathaba rufivena (Walker). SpringerPlus 2016, 5, 1944. [CrossRef]

21. Zhong, B.; Lv, C.; Qin, W. Effectiveness of the botanical insecticide azadirachtin against Tirathaba rufivena (Lepidoptera: Pyralidae). Fla Entomol. 2017, 100, 215-218. [CrossRef]

22. Venthur, H.; Zhou, J.-J. Odorant receptors and odorant-binding proteins as insect pest control targets: A comparative analysis. Front. Physiol. 2018, 9, 1163. [CrossRef] 
23. Karlsson, M.F.; Proffit, M.; Birgersson, G. Host-plant location by the Guatemalan potato moth Tecia solanivora is assisted by floral volatiles. Chemoecology 2017, 27, 187-198. [CrossRef]

24. Bai, P.H.; Wang, H.M.; Liu, B.S.; Li, M.; Liu, B.M.; Gu, X.S.; Tang, R. Botanical volatiles selection in mediating electrophysiological responses and reproductive behaviors for the fall webworm Moth Hyphantria cunea. Front. Physiol. 2020, 11, 486. [CrossRef]

25. Wei, H.S.; Qin, J.H.; Cao, Y.Z.; Li, K.B.; Yin, J. Two classic OBPs modulate the responses of female Holotrichia oblita to three major ester host plant volatiles. Insect Mol. Biol. 2021, 30, 390-399. [CrossRef]

26. Zhang, M.; Huang, Y.L.; Song, F.; Chen, W.J.; Zhao, S.L.; Deng, F.M. Analysis of aromatic components in areca inflorescence by SPME coupled with GC-MS. Chin. J. Trop. Crops 2014, 35, 1244-1249.

27. Li, Z.D. Preliminary study on the grading of ovarian development characteristics and volatile activity of betel flower to the Tirathaba rufivena Walker. Master's Thesis, Hainan University, Haikou, China, 2021; pp. 20-23.

28. Robert, X.; Gouet, P. Deciphering key features in protein structures with the new ENDscript server. Nucleic Acids Res. 2014, 42, W320-W324. [CrossRef]

29. Liu, H.; Duan, H.; Wang, Q.; Xiao, Y.; Wang, Q.; Xiao, Q.; Sun, L.; Zhang, Y. Key Amino Residues Determining Binding Activities of the Odorant Binding Protein AlucOBP22 to Two Host Plant Terpenoids of Apolygus lucorum. J. Agric. Food Chem. 2019, 67, 5949-5956. [CrossRef]

30. Wang, C.Q.; Li, J.Q.; Li, E.T.; Nyamwasa, I.; Li, K.B.; Zhang, S.; Peng, Y.; Wei, Z.J.; Yin, J. Molecular and functional characterization of odorant-binding protein genes in Holotrichia oblita Faldermann. Int. J. Biol. Macromol. 2019, 136, 359-367. [CrossRef]

31. Xu, Y.L.; He, P.; Zhang, L.; Fang, S.Q.; Dong, S.L.; Zhang, Y.J.; Li, F. Large-scale identification of odorant-binding proteins and chemosensory proteins from expressed sequence tags in insects. BMC Genom. 2009, 10, 632. [CrossRef] [PubMed]

32. Zhou, J.-J.; Zhang, G.-A.; Huang, W.; Birkett, M.A.; Field, L.M.; Pickett, J.A.; Pelosi, P. Revisiting the odorant-binding protein LUSH of Drosophila melanogaster: Evidence for odour recognition and discrimination. FEBS Lett. 2004, 558, 23-26. [CrossRef]

33. Ullah, R.M.K.; Quershi, S.R.; Adeel, M.M.; Abdelnabby, H.; Waris, M.I.; Duan, S.G.; Wang, M.Q. An odorant binding protein (SaveOBP9) involved in chemoreception of the wheat aphid Sitobion avenae. Int. J. Mol. Sci. 2020, 21, 8331. [CrossRef] [PubMed]

34. Yao, R.; Zhao, M.; Zhong, L.; Li, Y.; Li, D.; Deng, Z.; Ma, X. Characterization of the binding ability of the odorant binding protein BminOBP9 of Bactrocera minax to citrus volatiles. Pest. Manag. Sci. 2021, 77, 1214-1225. [CrossRef] [PubMed]

35. Rizwangul, A.; Cao, Y.; Zhang, S.; Yin, J.; Li, X.; Li, K. Feeding preference and taxis behavior of adult Holotrichia oblita (Coleoptera: Scarabaeidae) on three plants. Acta Entomol. Sin. 2018, 61, 585-595.

36. Huang, G.Z.; Liu, J.T.; Zhou, J.J.; Wang, Q.; Dong, J.Z.; Zhang, Y.J.; Li, X.C.; Li, J.; Gu, S.H. Expressional and functional comparisons of two general odorant binding proteins in Agrotis ipsilon. Insect Biochem. Mol. Biol. 2018, 98, 34-47. [CrossRef]

37. Zhang, Y.L.; Fu, X.B.; Cui, H.C.; Zhao, L.; Yu, J.Z.; Li, H.L. Functional Characteristics, Electrophysiological and antennal immunolocalization of general odorant-binding protein 2 in tea geometrid, Ectropis obliqua. Int. J. Mol. Sci. 2018, 19, 875. [CrossRef] [PubMed]

38. Ishida, Y.; Ishibashi, J.; Leal, W.S. Fatty acid solubilizer from the oral disk of the blowfly. PLoS ONE 2013, 8, e51779. [CrossRef]

39. Chen, D.; Zhang, L.; Xing, Z.; Lei, Z. Identification and function of the OBP13 protein from the leafminer (Liriomyza sativae). Sci. Agric. Sin. 2018, 51, 893-904.

40. Kyte, J.; Doolittle, R.F. A simple method for displaying the hydropathic character of a protein. J. Mol. Biol. 1982, 157, 105-132. [CrossRef]

41. Ahmed, T.; Zhang, T.; Wang, Z.; He, K.; Bai, S. Molecular cloning, expression profile, odorant affinity, and stability of two odorant-binding proteins in Macrocentrus cingulum Brischke (Hymenoptera: Braconidae). Arch. Insect Biochem. Physiol. 2017, 94, e21374. [CrossRef]

42. Ma, L.; Li, Z.; Zhang, W.; Cai, X.; Luo, Z.; Zhang, Y.; Chen, Z. The Odorant Binding Protein 6 Expressed in Sensilla Chaetica Displays Preferential Binding Affinity to Host Plants Volatiles in Ectropis obliqua. Front. Physiol. 2018, 9, 534. [CrossRef]

43. Ju, Q.; Qu, M.J.; Wang, Y.; Jiang, X.J.; Li, X.; Dong, S.L.; Han, Z.J. Molecular and biochemical characterization of two odorantbinding proteins from dark black chafer, Holotrichia parallela. Genome 2012, 55, 537-546. [CrossRef]

44. Lautenschlager, C.; Leal, W.S.; Clardy, J. Bombyx mori pheromone-binding protein binding nonpheromone ligands: Implications for pheromone recognition. Structure 2007, 15, 1148-1154. [CrossRef] 